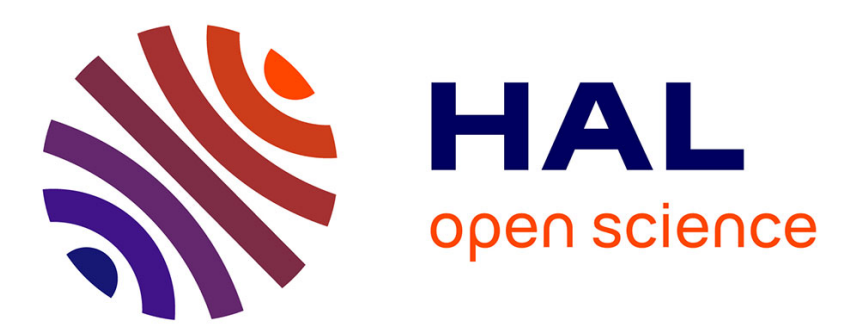

\title{
Robust tracking scheme for an experimental quadrotor
} L A Blas, M Bonilla, S Salazar, Michel Malabre, V Azhmyakov

\section{To cite this version:}

L A Blas, M Bonilla, S Salazar, Michel Malabre, V Azhmyakov. Robust tracking scheme for an experimental quadrotor. European Control Conference ECC21, Jun 2021, Rotterdam, Netherlands. hal-03174742

\section{HAL Id: hal-03174742 \\ https://hal.science/hal-03174742}

Submitted on 19 Mar 2021

HAL is a multi-disciplinary open access archive for the deposit and dissemination of scientific research documents, whether they are published or not. The documents may come from teaching and research institutions in France or abroad, or from public or private research centers.
L'archive ouverte pluridisciplinaire HAL, est destinée au dépôt et à la diffusion de documents scientifiques de niveau recherche, publiés ou non, émanant des établissements d'enseignement et de recherche français ou étrangers, des laboratoires publics ou privés. 


\title{
Robust tracking scheme for an experimental quadrotor
}

\author{
L.A. Blas ${ }^{1}$, M. Bonilla ${ }^{2}$, S. Salazar ${ }^{3}$, M. Malabre ${ }^{4}$ and V. Azhmyakov ${ }^{5}$
}

\begin{abstract}
In this paper, we present a robust tracking scheme for an experimental quadrotor, with outdoor real-time implementation. This control scheme is based on the robust structural feedback linearization scheme, presented in [2] . This control scheme has the advantage of combining the classical linear control techniques with the sophisticated robust control techniques. This control scheme is specially ad hoc for unmanned aerial vehicles, where it is important not only to reject the actual nonlinearities and the unexpected changes of the structure, but also to look for the simplicity and effectiveness of the control scheme.
\end{abstract}

keywords Quadrotor aircraft, unmanned aerial vehicle (UAV), trajectory tracking, non-standard feedback linearization, nonlinearities rejection.

\section{INTRODUCTION}

In [2], we have presented a synthesis procedure of the recently robust linear control scheme proposed in [3], which is based on failure detection techniques. Such a linear control approach is intended to reject linearly structured uncertainties, which are treated as failure signals affecting the systems dynamics. In this paper we present some necessary adjustments for tracking some prescribed trajectory in the $\mathrm{x}$ y plane, in outdoor real-time experiments. In Section II, we recall some preliminaires results. In Section III, we present a drift-free estimator for overcomimng the drift due to the integration of wind noises. In Section IV, we present outdoor real-time experiments obtained when the quadrotor tracks a circular trajectory in the $x-y$ plane. And in Section V, we conclude.

\section{PRELIMINAIRES}

\section{A. Quadrotor Description}

In this paper we deal with the quadrotor laboratory prototype shown in Figure 1.

\footnotetext{
${ }^{1}$ Luis Ángel Blas was a Ph.D. student sponsored by CONACyT México at CINVESTAV-IPN, Control Automático, México. anghelblas@gmail.com

${ }^{2}$ Moisés Bonilla is with CINVESTAV-IPN, Control Automático, UMI 3175 CINVESTAV-CNRS. A.P. 14-740. México 07000. mbonillaecinvestav.mx

${ }^{2}$ Moisés Bonilla is with CINVESTAV-IPN, Control Automático, UMI 3175 CINVESTAV-CNRS. A.P. 14-740. México 07000. mbonillaecinvestav.mx

${ }^{3}$ Sergio Salazar is with CINVESTAV-IPN, Sistemas Autónomos de Navegación Aérea Y Submarina, UMI 3175 CINVESTAV-CNRS. A.P. 14740. México 07000. sergio.salazar. cruz@gmail.com

4 This work was done while Michel Malabre was with CNRS, LS2N, UMR 6004, Nantes, France. Michel.Malabreds $2 n$.fr

${ }^{5}$ Vadim Azhmyakov is with Facultad de Ingeniera y Ciencias Básicas, Universidad Central, Bogotá, Colombia, and with Institut für Theoretische Informatik, Mathematik und Operations Research, Universität der Bundeswehr München, München, Germany. vazhmyakodeafit.edu.co
}

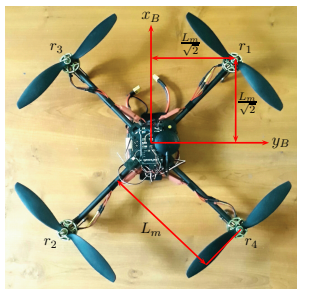

(a)

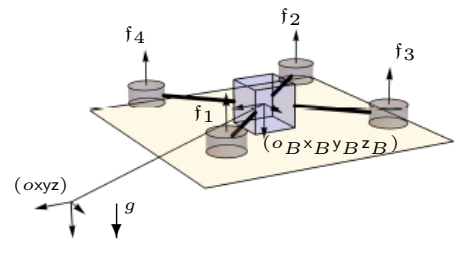

(b)
Fig. 1. (a) Quadrotor. (b) Schematic diagram.

The motion of the quadrotor is referred to a fixed orthogonal axis set (earth axes) (oxyz), where oz points vertically down along the gravity vector ${ }^{1}\left[\begin{array}{lll}0 & 0 & g\end{array}\right]^{T}$, and the origin $o$ is located at the desired height $\bar{z}$, above the ground level. $\phi$, $\theta$ and $\psi$ are the Euler angles, roll, pitch and yaw, measured respectively over the axis $o_{B} \mathrm{x}_{B}, o_{B} \mathrm{y}_{B}$ and $o_{B} z_{B}$; where $\left(o_{B} \mathrm{x}_{B} \mathrm{y}_{B} \mathrm{z}_{B}\right)$ is the body axis system, with its origin $o_{B}$ fixed at the centre of gravity of the quadrotor; see Figure 1. The total mass is $M_{q}$, the moments of inertia about axis $o x$, $o y$ and $o z$ are $\mathcal{I}_{x x}, \mathcal{I}_{y y}$ and $\mathcal{I}_{z z}$, and the distance of each rotor with respect to the centre of gravity of the quadrotor is denoted by $L_{m}:{ }^{2}$

$$
\begin{gathered}
M_{q}=1.36[\mathrm{~kg}], \mathcal{I}_{x x}=0.0134\left[\mathrm{~kg} \mathrm{~m}^{2}\right], \mathcal{I}_{y y}=0.0140\left[\mathrm{~kg} \mathrm{~m}^{2}\right], \\
\mathcal{I}_{z z}=0.0256\left[\mathrm{~kg} \mathrm{~m}^{2}\right], L_{m}=0.245[\mathrm{~m}]
\end{gathered}
$$

In [1], we have shown that the quadrotor is represented by the state space representations:

$\mathrm{d} \mathbf{x}_{i} / \mathrm{d} t=\mathbf{A}_{i} \mathbf{x}_{i}+\mathbf{B}_{i} u_{i}+\mathbf{S}_{i} \mathbf{q}_{o i}, \mathbf{y}_{i}=\mathbf{C}_{i} \mathbf{x}_{i}, i \in\{\mathrm{x}, \mathrm{y}, \mathrm{z}, \psi\}$,

$\mathbf{A}_{\times}=\left[\begin{array}{rrrr}0 & 1 & 0 & 0 \\ 0 & 0 & -g & 0 \\ 0 & 0 & 0 & 1 \\ 0 & 0 & 0 & 0\end{array}\right], \mathbf{A}_{\mathrm{y}}=\left[\begin{array}{llll}0 & 1 & 0 & 0 \\ 0 & 0 & g & 0 \\ 0 & 0 & 0 & 1 \\ 0 & 0 & 0 & 0\end{array}\right]$,

$\mathbf{B}_{\mathrm{x}}=\mathcal{I}_{y y}^{-1} \mathbf{B}_{4}, \mathbf{B}_{\mathrm{y}}=\mathcal{I}_{x x}^{-1} \mathbf{B}_{4}$,

$\mathbf{B}_{4}=\left[\begin{array}{l}0 \\ 0 \\ 0 \\ 1\end{array}\right], \mathbf{S}_{\mathrm{x}}=\mathbf{S}_{\mathrm{y}}=\left[\begin{array}{ll}0 & 0 \\ 1 & 0 \\ 0 & 0 \\ 0 & 1\end{array}\right], \mathbf{C}_{\mathrm{x}}=\mathbf{C}_{\mathrm{y}}=\left[\begin{array}{l}1 \\ 0 \\ 0 \\ 0\end{array}\right]^{T}$,

$\mathbf{A}_{\mathbf{z}}=\mathbf{A}_{\psi}=\left[\begin{array}{ll}0 & 1 \\ 0 & 0\end{array}\right], \mathbf{B}_{\mathbf{z}}=M_{q}^{-1} \mathrm{~B}_{2}, \mathbf{B}_{\psi}=\mathcal{I}_{z z}^{-1} \mathrm{~B}_{2}$,

$\mathbf{S}_{\mathbf{z}}=\mathbf{S}_{\psi}=\mathbf{B}_{2}, \mathbf{B}_{2}=\left[\begin{array}{l}0 \\ 1\end{array}\right], \mathbf{C}_{\mathbf{z}}=\mathbf{C}_{\psi}=\left[\begin{array}{l}1 \\ 0\end{array}\right]^{T}$.

${ }^{1}$ We take the value: $g=9.81\left[\mathrm{~m} \mathrm{~s}^{-2}\right]$.

${ }^{2}$ Since the quadrotor is mechanically symmetric its cross inertia are zero. 


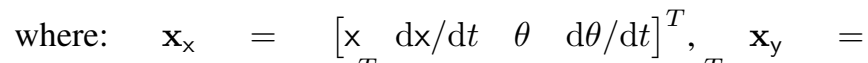
$\left[\begin{array}{llll}\mathrm{y} & \mathrm{dy} / \mathrm{d} t & \phi & \mathrm{d} \phi / \mathrm{d} t\end{array}\right]^{T}, \quad \mathbf{x}_{\mathrm{z}}=\left[\begin{array}{ll}\mathrm{z} & \mathrm{d} z / \mathrm{d} t\end{array}\right]^{T}, \quad \mathbf{x}_{\psi}=$ $\left[\begin{array}{ll}\psi & \mathrm{d} \psi / \mathrm{d} t\end{array}\right]^{T}, \mathbf{q}_{\mathrm{ox}}=\left[\begin{array}{ll}q_{\times} & q_{\theta}\end{array}\right]^{T}, \mathbf{q}_{\text {oy }}=\left[\begin{array}{ll}q_{\mathrm{y}} & q_{\phi}\end{array}\right]^{T}, \mathbf{q}_{\mathrm{oz}}=$ $q_{\mathrm{z}}, \mathbf{q}_{o \psi}=q_{\psi}$. The control actions, $u_{\mathrm{x}}, u_{\mathrm{y}}, u_{\mathrm{z}}$ and $u_{\psi}$, are related with the thrusters of the four rotors, $\mathfrak{f}_{1}, \mathfrak{f}_{2}, \mathfrak{f}_{3}$ and $\mathfrak{f}_{4}$, by means of an invertible real constant matrix ( $c f$. Section 5 of [1]). The nonlinear signals, $q_{\times}, q_{\mathrm{y}}, q_{\mathrm{z}}, q_{\phi}, q_{\theta}$ and $q_{\psi}$, are:

$$
\begin{gathered}
{\left[\begin{array}{c}
q_{\mathrm{x}} \\
q_{\mathrm{y}} \\
q_{\mathrm{z}}
\end{array}\right]=\left[\begin{array}{c}
\theta-q_{\mathrm{xx}} \\
-\phi-q_{\mathrm{yy}} \\
-q_{\mathrm{zz}}
\end{array}\right] g+\frac{1}{M_{q}}\left[\begin{array}{c}
q_{\mathrm{xx}} \\
q_{\mathrm{yy}} \\
q_{\mathrm{zz}}
\end{array}\right] u_{\mathrm{z}},} \\
{\left[\begin{array}{c}
q_{\phi} \\
q_{\theta} \\
q_{\psi}
\end{array}\right]=\left(\mathbb{J}^{-1}(\eta)-\mathbb{J}^{-1}(0)\right) \tau-\mathbb{J}^{-1}(\eta) C(\eta, \mathrm{d} \eta / \mathrm{d} t) \frac{\mathrm{d} \eta}{\mathrm{d} t},}
\end{gathered}
$$

where $\eta=\left[\begin{array}{lll}\phi & \theta & \psi\end{array}\right]^{T}, \tau=\left[\begin{array}{lll}u_{\mathrm{y}} & u_{\mathrm{x}} & u_{\psi}\end{array}\right]^{T}, \mathbb{J}$ and $C(\eta, \mathrm{d} \eta / \mathrm{d} t)$ are the inertial and the Coriolis matrices, defined in Appendix A of [1], and:

$$
\begin{aligned}
& q_{\mathrm{xx}}=\cos (\phi) \sin (\theta) \cos (\psi)+\sin (\phi) \sin (\psi), \\
& q_{\mathrm{yy}}=\cos (\phi) \sin (\theta) \sin (\psi)-\sin (\phi) \cos (\psi), \\
& q_{\mathrm{zz}}=\cos (\phi) \cos (\theta)-1 .
\end{aligned}
$$

\section{B. Locally Stabilizing Feedback}

In the fifth Section of [2], we have used the LQR technique in order to locally stabilize $(2.2)-(2.4)^{3}$. For this, we have solved the Algebraic Riccati Equations (ARE):

$$
\mathbf{A}_{i}^{T} \mathbf{P}_{i}+\mathbf{P}_{i} \mathbf{A}_{i}-\mathbf{P}_{i} \mathbf{B}_{i}\left(\rho_{i} \mathrm{I}\right)^{-1} \mathbf{B}_{i}^{T} \mathbf{P}_{i}+\mathbf{Q}_{i}=0,
$$

where $i \in\{\mathrm{x}, \mathrm{y}, \mathrm{z}, \psi\}$, and with:

$$
\begin{gathered}
\mathbf{Q}_{x}=\mathbf{Q}_{\mathbf{y}}=900\left[\begin{array}{cccc}
1 & 0 & 0 & 0 \\
0 & 0 & 0 & 0 \\
0 & 0 & 1 & 0 \\
0 & 0 & 0 & 2.25
\end{array}\right], \\
\mathbf{Q}_{\mathbf{z}}=\left[\begin{array}{cc}
1 & 0 \\
0 & 0.23
\end{array}\right], \mathbf{Q}_{\psi}=\left[\begin{array}{cc}
1 & 0 \\
0 & 0.6
\end{array}\right],
\end{gathered}
$$$$
\left(\rho_{\mathrm{x}}, \rho_{\mathrm{y}}, \rho_{\mathrm{z}}, \rho_{\psi}\right)=(1,1,1 / 19600,1 / 12100) .
$$

Solving (2.8) for matrices (2.3) and (2.4), with the choices (2.9) and (2.10), we have obtained $(i \in\{x, y, z, \psi\})$ :

$$
\begin{aligned}
& \mathbf{u}_{i}=\mathbf{F}_{i} \mathbf{x}_{i}+\overline{\mathbf{u}}_{i}, \quad i \in\{\mathrm{x}, \mathrm{y}\}, \\
& \mathbf{u}_{i}=\mathbf{F}_{i}\left(\mathbf{x}_{i}-\overline{\mathbf{x}}_{i}\right), i \in\{\mathbf{z}, \psi\}, \quad \overline{\mathbf{x}}_{\mathbf{z}}=\left[\begin{array}{cc}
\overline{\mathbf{z}} & 0
\end{array}\right]^{T}, \overline{\mathbf{x}}_{\psi}=0, \\
& \mathbf{F}_{\mathrm{x}}=\left[\begin{array}{llll}
30 & 32.4264 & -171.9158 & -45.0535
\end{array}\right], \\
& \mathbf{F}_{\mathrm{y}}=\left[\begin{array}{llll}
-30 & -32.4260 & -171.9116 & -45.0512
\end{array}\right], \\
& \mathbf{F}_{\mathrm{z}}=\left[\begin{array}{ll}
-140 & -69.92
\end{array}\right], \quad \mathbf{F}_{\psi}=\left[\begin{array}{ll}
-110 & -85.2387
\end{array}\right] .
\end{aligned}
$$

With the state feedbacks (2.11), (2.12) and (2.13), we get the following spectra and characteristic polynomials:

$$
\begin{aligned}
& \left.\Lambda_{\mathbf{A}_{\mathbf{F}_{\mathbf{x}}}}=\{-3214,-1.911,-0.9536 \pm 1.585\}\right\}, \\
& \left.\Lambda_{\mathbf{A}_{\mathbf{F}_{\mathbf{y}}}}=\{-3358,-1.911,-0.9536 \pm 1.585\}\right\}, \\
& \Lambda_{\mathbf{A}_{\mathbf{F}_{\mathbf{z}}}}=\{-2.087,-49.32\}, \quad \Lambda_{\mathbf{A}_{\mathbf{F}_{\psi}}}=\{-1.291,-3328\},
\end{aligned}
$$

\footnotetext{
${ }^{3}$ Recall the celebrated Stability Principle of the First Approximation (see for example [8]).
}

$\pi_{x_{i}}(\mathrm{~s})=\operatorname{det}\left(\mathrm{sI}-\mathbf{A}_{\mathbf{F}_{i}}\right)=\mathrm{s}^{4}+a_{i, 1} \mathrm{~s}^{3}+a_{i, 2} \mathrm{~s}^{2}+a_{i, 3} \mathrm{~s}+a_{i, 4}$,

where: $\mathbf{A}_{\mathbf{F}_{i}} \doteq \mathbf{A}_{i}+\mathbf{B}_{i} \mathbf{F}_{i}, i \in\{\mathrm{x}, \mathrm{y}, \mathrm{z}, \psi\}$.

\section{Nonlinear Uncertainty Signal Estimator}

In [1], we have shown that with the change of variable: ${ }^{4}$

$$
\zeta_{i}=\mathbf{x}_{i}+\mathbf{M}_{i}\left(\mathbf{S}_{i}+\sum_{j=1}^{n-1} \mathbf{M}_{i}^{j} \mathbf{S}_{i} \mathrm{~d}^{j} / \mathrm{d} t^{j}\right) \mathbf{q}_{o i}(x, u),
$$

the state space descriptions (2.2), fedback by (2.11) and (2.13), take the form $(i \in\{x, y\})$ :

$$
\mathrm{d} \zeta_{i} / \mathrm{d} t=\mathbf{A}_{\mathbf{F}_{i}} \zeta_{i}+\mathbf{B}_{i}\left(\overline{\mathbf{u}}_{i}+\mathbf{q}_{* i}\left(\mathbf{x}_{i}, \mathbf{u}_{i}\right)\right), \quad \mathbf{y}_{i}=\mathbf{C}_{i} \zeta_{i},
$$

where the nonlinear uncertainty signals, $\mathbf{q}_{* i}$, are:

$\mathbf{q}_{* i}\left(\left(\mathbf{x}_{i}, \mathbf{u}_{i}\right)=\mathbf{X}_{i}\left(\mathbf{S}_{i}+\sum_{j=1}^{n-1} \mathbf{M}_{i}^{j} \mathbf{S}_{i} \mathrm{~d}^{j} / \mathrm{d} t^{j}\right) \mathbf{q}_{o i}\left(\mathbf{x}_{i}, \mathbf{u}_{i}\right)\right.$.

The matrices $\mathbf{M}_{i}$ and $\mathbf{X}_{i}$, are solutions of the matrix equtions:

$$
\mathbf{A}_{\mathbf{F}_{i}} \mathbf{M}_{i}+\mathbf{B}_{i} \mathbf{X}_{i}=\mathrm{I} .
$$

In Section V.B.2 of [2], we have synthesized the following nonlinear uncertainty signals observers for (2.2), with $i \in$ $\{x, y\}$ and the matrices (2.3):

$$
\begin{aligned}
\mathrm{d} \mathbf{w}_{i} / \mathrm{d} t & =\left(\mathbf{A}_{\mathbf{K}_{i}}+\mathbf{B}_{i} \mathbf{G}_{i}^{\ell} \mathbf{C}_{i}\right) \mathbf{w}_{i}-\left(\mathbf{K}_{i}+\mathbf{B}_{i} \mathbf{G}_{i}^{\ell}\right) \mathbf{y}_{i}, \\
\overline{\mathbf{u}}_{i} & =\mathbf{G}_{i}^{\ell}\left(\mathbf{C}_{i} \mathbf{w}_{i}-\mathbf{y}_{i}\right),
\end{aligned}
$$

where: $\mathbf{A}_{\mathbf{K}_{i}}=\mathbf{A}_{\mathbf{F}_{i}}+\mathbf{K}_{i} \mathbf{C}_{i}$ and $\mathbf{G}_{i}^{\ell}=-\left(\mathbf{C}_{i} \mathbf{A}_{\mathbf{K}_{i}}^{-1} \mathbf{B}_{i}\right)^{\ell} \in$ $\mathbb{R}^{1 \times 1}, i \in\{\mathrm{x}, \mathrm{y}\}$, and (cf. [6]):

$$
\begin{aligned}
& \mathbf{A}_{\mathbf{F}_{i}}=\left[\begin{array}{cccc}
0 & 0 & 0 & -a_{i, 4} \\
1 & 0 & 0 & -a_{i, 3} \\
0 & 1 & 0 & -a_{i, 2} \\
0 & 0 & 1 & -a_{i, 1}
\end{array}\right], \mathbf{K}_{i}=\left[\begin{array}{c}
a_{i, 4}-a_{o, 4} \\
a_{i, 3}-a_{o, 3} \\
a_{i, 2}-a_{o, 2} \\
a_{i, 1}-a_{o, 1}
\end{array}\right], \\
& \mathbf{C}_{i}=\left[\begin{array}{llll}
0 & 0 & 0 & 1
\end{array}\right], \mathbf{B}_{i}=\left[\begin{array}{cccc}
\frac{k_{i}}{b_{i}} & 0 & 0 & 0
\end{array}\right]^{T}, \\
& \mathbf{G}_{i}^{\ell}=-\left(\mathbf{C}_{i}\left(\mathbf{A}_{\mathbf{F}_{i}}+\mathbf{K}_{i} \mathbf{C}_{i}\right)^{-1} \mathbf{B}_{i}\right)^{\ell}=\left(a_{o, 4} b_{i}\right) / k_{i},
\end{aligned}
$$

where: $i \in\{\mathrm{x}, \mathrm{y}\},\left(a_{i, 1}, a_{i, 2}, a_{i, 3}, a_{i, 4}\right)$ are the coefficients of the polynomials $(2.14)$, and: $\left(k_{\times}, b_{\times}\right)=\left(-g, \mathcal{I}_{y y}\right)$ and $\left(k_{\mathrm{y}}, b_{\mathrm{y}}\right)=\left(g, \mathcal{I}_{x x}\right)$.

Let us note that the output signals of the nonlinear uncertainty signals observers (2.19) are the negative estimations of the nonlinear uncertainty signals $\mathbf{q}_{* i}$, namely: $\overline{\mathbf{u}}_{i}=-\hat{\mathbf{q}}_{* i}$.

The transfer functions $\mathrm{F}_{C L_{i}}(\mathrm{~s})$ of the closed loop system are $\left(\mathbf{y}_{i} \text { v.s. } \mathbf{q}_{* i}\right)^{5}$

$$
\mathrm{F}_{C L_{i}}(\mathrm{~s})=\mathrm{F}_{\zeta_{i}}(\mathrm{~s}) \mathrm{G}_{\mathrm{HPF}}(\mathrm{s}),
$$

where:

$$
\mathrm{F}_{\zeta_{i}}(\mathrm{~s})=\mathbf{C}_{i}\left(\mathrm{sI}-\mathbf{A}_{\mathbf{F}_{i}}\right)^{-1} \mathbf{B}_{i}=\frac{k_{i}}{b_{i} \pi_{x_{i}}(\mathrm{~s})},
$$

${ }^{4} C f$. Lemma 1 and Theorem 1 of [2].

${ }^{5}$ See Section IV.D of [2]. 


$$
\begin{aligned}
\mathrm{G}_{\mathrm{HPF}}(\mathrm{s}) & =1-\mathrm{F}_{e}(\mathrm{~s})=\mathbf{G}_{i}^{\ell} \mathbf{C}_{i}\left(\mathrm{sI}-\mathbf{A}_{\mathbf{K}_{i}}\right)^{-1} \mathbf{B}_{i} \\
& =1-a_{o, 4} / \pi_{e_{i}}(\mathrm{~s})=\mathrm{s} \bar{\pi}_{w}(\mathrm{~s}) / \pi_{e}(\mathrm{~s}),
\end{aligned}
$$

and:

$$
\begin{aligned}
\pi_{e}(\mathrm{~s})= & \operatorname{det}\left(\mathrm{sI}-\mathbf{A}_{\mathbf{K}_{i}}\right) \\
= & \mathrm{s}^{4}+a_{o, 1} \mathrm{~s}^{3}+a_{o, 2} \mathrm{~s}^{2}+a_{o, 3} \mathrm{~s}+a_{o, 4}, \\
\pi_{w}(\mathrm{~s})= & \operatorname{det}\left(\mathrm{sI}-\left(\mathbf{A}_{\mathbf{K}_{i}}+\mathbf{B}_{i} \mathbf{G}_{i}^{\ell} \mathbf{C}_{i}\right)\right) \\
= & \mathrm{s}\left(\mathrm{s}^{3}+a_{o, 1} \mathrm{~s}^{2}+a_{o, 2} \mathrm{~s}+a_{o, 3}\right), \\
& \pi_{e}(\mathrm{~s})=\mathrm{s} \bar{\pi}_{w}(\mathrm{~s})+a_{o, 4} .
\end{aligned}
$$

Following a root locus procedure, in Section V.B.1 of [2] we have deduced:

$$
\begin{gathered}
\mathrm{s} \bar{\pi}_{w}(\mathrm{~s})=\mathrm{s}\left(\mathrm{s}+4.75 \varrho_{c}\right)\left(\mathrm{s}+4 \varrho_{c}\right)\left(\mathrm{s}+3.5 \varrho_{c}\right), \\
\pi_{e}(\mathrm{~s})=\left(\mathrm{s}+\varrho_{c}\right)^{2}\left(\mathrm{~s}^{2}+10.25 \varrho_{c} \mathrm{~s}+28.125 \varrho_{c}^{2}\right), \\
a_{o, 4}=28.125 \varrho_{c}^{4},
\end{gathered}
$$

and that the cutoff frequency $\omega_{c}$ of the high-pass filter

$$
\mathrm{G}_{\mathrm{HPF}}(\jmath \omega)=\frac{\left(\omega^{2}-a_{o, 2}\right) \omega^{2}-\jmath\left(a_{o, 1} \omega^{2}-a_{o, 3}\right) \omega}{\left(\left(\omega^{2}-a_{o, 2}\right) \omega^{2}+a_{o, 4}\right)-\jmath\left(a_{o, 1} \omega^{2}-a_{o, 3}\right) \omega}
$$

is:

$\omega_{c}=\sqrt{a_{o, 2} / 2} \sqrt{1-\sqrt{1-2 a_{o, 4} / a_{o, 2}^{2}}}=0.5339 \varrho_{c}[\mathrm{rad} / \mathrm{s}]$.

We then have to adjust $\varrho_{c}$ sufficiently higher than the bandwidth $\mathcal{B}_{\mathcal{W}}$ of the Fourier transform of $q_{* i}, \mathcal{F}\left\{q_{* i}\right\}$, $i \in\{\mathrm{x}, \mathrm{y}\}$. Experimentally, we have found that $\varrho_{c}=18$ is a good choice for our laboratory prototype, thus:

$\pi_{e}(\mathrm{~s})=\mathrm{s}^{4}+220.5 \mathrm{~s}^{3}+16078.5 \mathrm{~s}^{2}+387828 \mathrm{~s}+2952450$.

\section{DRIFT-FREE ESTIMATOR}

The nonlinear uncertainty signal estimator (2.19) has a good performance when the quadrotor is in hover flying.

However, when we wish that the quadrotor tracks some prescribed trajectory in the $x-y$ plane, sometimes the quadrotor suffers a drift phenomenon. Doing a frequency analysis of the recorded $x$ and $y$ signals in hover flying (in outdoor realtime experiments), we have found that the signal disturbance is well approximated by:

$$
\begin{array}{r}
\mathrm{d}(t)=0.009 \sin (0.85 t)+0.0115 \eta_{d}(t)+0.0073 ; \\
\eta_{d}(t)=\sin (2.1 \pi(\sin (2 \pi t / T)+1)),
\end{array}
$$

with $2 \pi / T==.4$. Analyzing (3.1), we deduce: (i) The constant term 0.0073 is easily compensated by the integral term of the nonlinear uncertainty estimator, see (2.25). (ii) The sinusoidal term $0.009 \sin (0.85 t)$ is certainly attenuated by the high-pass filter $\mathrm{G}_{\mathrm{HPF}}(\mathrm{s})$, see (2.21) and (2.23). Then, the $\eta_{d}(t)$ term is the cause of the drift phenomenon.

In order to overcome the quadrotor drift due to the integration of wind noises like (3.2), we have proceeded as in [4] and [5]; that is to say, we have shifted the pole at the origin of (2.19), slightly into the left-half complex plane ( $c f$.
(2.25)). For this, we have reduced lightly the static gain $\mathbf{G}_{i}^{\ell}$ of the nonlinear uncertainty signal estimator (2.19), namely:

$$
\begin{aligned}
\mathrm{d} \mathbf{w}_{d f, i} / \mathrm{d} t= & \left(\mathbf{A}_{\mathbf{K}_{i}}+(1-\epsilon) \mathbf{B}_{i} \mathbf{G}_{i}^{\ell} \mathbf{C}_{i}\right) \mathbf{w}_{d f, i} \\
& -\left(\mathbf{K}_{i}+(1-\epsilon) \mathbf{B}_{i} \mathbf{G}_{i}^{\ell}\right) \mathbf{y}_{i}, \\
\overline{\mathbf{u}}_{d f, i}= & (1-\epsilon) \mathbf{G}_{i}^{\ell}\left(\mathbf{C}_{i} \mathbf{w}_{d f, i}-\mathbf{y}_{i}\right),
\end{aligned}
$$

where $\epsilon$ is a sufficiently small positive constant and $i \in$ $\{x, y\}$.

Let us note that the output signals of the drift-free estimators (3.3) are $-(1-\epsilon)$ times the nonlinear uncertainty signals $\mathbf{q}_{* i}$, namely: $\overline{\mathbf{u}}_{i}=-(1-\epsilon) \hat{\mathbf{q}}_{* i}$.

Now, the characteristic polynomial of $\left(\mathbf{A}_{\mathbf{K}_{i}}+(1-\right.$ $\epsilon) \mathbf{B}_{i} \mathbf{G}_{i}^{\ell} \mathbf{C}_{i}$ ) has no roots at the origin (recall (2.20) and $c f$. (2.25)):

$$
\begin{array}{r}
\pi_{w_{d f}}(\mathrm{~s})=\operatorname{det}\left(\mathrm{sI}-\left(\mathbf{A}_{\mathbf{K}_{i}}+(1-\epsilon) \mathbf{B}_{i} \mathbf{G}_{i}^{\ell} \mathbf{C}_{i}\right)\right) \\
\pi_{w_{d f}}(0)=\operatorname{det} \mathbf{A}_{\mathbf{K}_{i}} \operatorname{det}\left(-1-(1-\epsilon) \mathbf{C}_{i} \mathbf{A}_{\mathbf{K}_{i}}^{-1} \mathbf{B}_{i} \mathbf{G}_{i}^{\ell}\right) \\
=-\epsilon \neq 0 .
\end{array}
$$

\section{A. Closed loop system}

Applying (3.3) to (2.16), we get the closed loop system represented by ( $c f .(4.16)$ and (4.17) of [2]):

$$
\begin{gathered}
\frac{\mathrm{d}}{\mathrm{d} t}\left[\begin{array}{c}
\mathbf{e}_{d f, i} \\
\zeta_{i}
\end{array}\right]=\mathbf{A}_{C L_{d f, i}}\left[\begin{array}{c}
\mathbf{e}_{d f, i} \\
\zeta_{i}
\end{array}\right]+\mathbf{B}_{C L_{i}} \mathbf{q}_{* i}, \\
\mathbf{y}_{i}=\mathbf{C}_{C L_{i}}\left[\begin{array}{c}
\mathbf{e}_{d f, i} \\
\zeta_{i}
\end{array}\right], \\
\mathbf{A}_{C L_{d f, i}}=\left[\begin{array}{c|c}
\mathbf{A}_{\mathbf{K}_{i}} & 0 \\
\hline(1-\epsilon) \mathbf{B}_{i} \mathbf{G}_{i}^{\ell} \mathbf{C}_{i} & \mathbf{A}_{\mathbf{F}_{i}}
\end{array}\right], \mathbf{B}_{C L_{i}}=\left[\begin{array}{r}
-\mathbf{B}_{i} \\
\hline \mathbf{B}_{i}
\end{array}\right], \\
\mathbf{C}_{C L_{i}}=\left[\begin{array}{l}
\left.0 \mid \mathbf{C}_{i}\right],
\end{array}\right.
\end{gathered}
$$

where: $\mathbf{e}_{d f, i}=\mathbf{w}_{d f, i}-\zeta_{i}$.

The transfer function of $\Sigma\left(\mathbf{A}_{C L_{d f, i}}, \mathbf{B}_{C L_{i}}, \mathbf{C}_{C L_{i}}\right)$ is (cf. (4.18) and (4.19) of [2]):

$$
\begin{aligned}
\mathrm{F}_{C L_{d f, i}}(\mathrm{~s}) & =\mathbf{C}_{C L_{i}}\left(\mathrm{sI}-\mathbf{C}_{C L_{i}}\right)^{-1} \mathbf{B}_{C L_{i}} \\
& =\mathrm{F}_{\zeta_{i}}(\mathrm{~s})\left(\mathrm{I}-\mathrm{F}_{\left.\mathbf{e}_{d f, i}(\mathrm{~s})\right)}\right. \\
\mathrm{F}_{\zeta_{i}}(\mathrm{~s}) & =\mathbf{C}_{i}\left(\mathrm{sI}-\mathbf{A}_{\mathbf{F}_{i}}\right)^{-1} \mathbf{B}_{i} \\
\mathrm{~F}_{\mathbf{e}_{d f, i}}(\mathrm{~s}) & =(1-\epsilon) \mathbf{G}_{i}^{\ell} \mathbf{C}_{i}\left(\mathrm{sI}-\mathbf{A}_{\mathbf{K}_{i}}\right)^{-1} \mathbf{B}_{i}
\end{aligned}
$$

together with (2.20).

From (3.3), (2.20) and (3.7), we get (recall (2.24) and $(2.26)):^{6}$

$$
\begin{aligned}
& \pi_{w_{d f}}(\mathrm{~s})=\mathrm{s} \bar{\pi}_{w}(\mathrm{~s})+\epsilon a_{o, 4}, \\
& \mathrm{~F}_{e_{d f, i}}(\mathrm{~s})=(1-\epsilon) a_{o, 4} / \pi_{e}(\mathrm{~s}),
\end{aligned}
$$

\section{B. Frequency response}

The closed loop transfer function (3.6) incorporates the high-pass filter (recall (3.9), (2.26) and (3.8)):

$$
\begin{gathered}
\mathrm{G}_{\mathrm{HFF}_{d f}}(\mathrm{~s})=1-\mathrm{F}_{e_{d f, i}}(\mathrm{~s})=1-\frac{(1-\epsilon) a_{o, 4}}{\pi_{e}(\mathrm{~s})} \\
=\frac{\mathrm{s} \bar{\pi}_{w}(\mathrm{~s})+\epsilon a_{o, 4}}{\pi_{e}(\mathrm{~s})} \\
{ }^{6} \pi_{w_{d f, i}}(\mathrm{~s})=\operatorname{det}\left(\mathrm{sI}_{4}-\left(\left(\mathbf{A}_{\mathbf{F}_{i}}+\mathbf{K}_{i} \mathbf{C}_{i}\right)+(1-\epsilon) \mathbf{B}_{i} \mathbf{G}_{i}^{\ell} \mathbf{C}_{i}\right)\right) .
\end{gathered}
$$




$$
\begin{aligned}
\mathrm{G}_{\mathrm{HPF}_{d f}}(\jmath \omega) & = \\
& \frac{\left(\left(\omega^{2}-a_{o, 2}\right) \omega^{2}+\varepsilon a_{o, 4}\right)-\jmath\left(a_{o, 1} \omega^{2}-a_{o, 3}\right) \omega}{\left(\left(\omega^{2}-a_{o, 2}\right) \omega^{2}+a_{o, 4}\right)-\jmath\left(a_{o, 1} \omega^{2}-a_{o, 3}\right) \omega} .
\end{aligned}
$$

The cutoff frequency $\omega_{c_{d f}}$ of the high-pass filter $\mathrm{G}_{\mathrm{HPF}_{d f}}(\jmath \omega)$ for $\epsilon=1 / 50$ is (recall (2.28) and $c f$. (2.31)): ${ }^{7}$

$$
\begin{aligned}
\omega_{c_{d f}} & =\sqrt{a_{o, 2} / 2} \sqrt{1-\sqrt{1-2(1+\epsilon) a_{o, 4} / a_{o, 2}^{2}}} \\
& =0.5392 \varrho_{c}[\mathrm{rad} / \mathrm{s}] .
\end{aligned}
$$

In Figure 2, we compare the Bode plots of $\mathrm{G}_{\mathrm{HPF}}(\jmath \omega),(2.30)$ and (2.28), and $\mathrm{G}_{\mathrm{HPF}_{d f}}(\jmath \omega),(3.11)$ and (2.28), with $\varrho_{c}=$ 18 and $\epsilon=1 / 50$. From Figure 2, we realize that the high frequencies behaviors are very similar, and the low frequencies are attenuated by the factor $\epsilon$. Now, there is no poles at the origin, avoiding in this way the drift phenomena due to the integration of wind noises like (3.2).

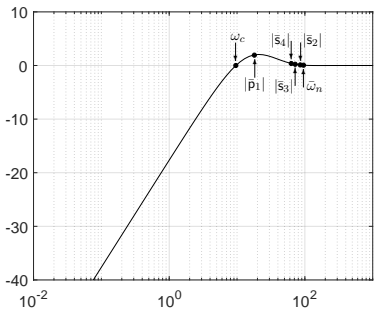

(a)

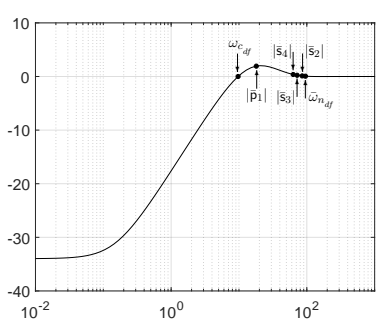

(b)
Fig. 2. (a) Bode plot of $\left|\mathrm{G}_{\mathrm{HPF}}(\jmath \omega)\right|_{d b}$, (2.30) and (2.28), with $\varrho_{c}=18$. $\left|\overline{\mathbf{p}}_{1}\right|=18,\left|\overline{\mathbf{s}}_{2}\right|=85.5,\left|\overline{\mathbf{s}}_{3}\right|=72,\left|\overline{\mathbf{s}}_{4}\right|=63, \bar{\omega}_{n}=95.46[\mathrm{rad} / \mathrm{s}]$, $\bar{\zeta}=0.966$ and $\omega_{c}=9.61[\mathrm{rad} / \mathrm{s}] ;\left|\mathrm{G}_{\mathrm{HPF}}(0)\right|=0$ and $\left|\mathrm{G}_{\mathrm{HPF}}(\infty)\right|=1$. (b) Bode plot of $\left|\mathrm{G}_{\mathrm{HPF}_{d f}}(\jmath \omega)\right|_{d b}$, (3.11) and (2.28), with $\varrho_{c}=18$ and $\epsilon$ $=1 / 50 .\left|\overline{\mathrm{p}}_{1 d f}\right|=18,\left|\overline{\mathbf{s}}_{2 d f}\right|=85.5,\left|\overline{\mathbf{s}}_{3 d f}\right|=72,\left|\overline{\mathbf{s}}_{4 d f}\right|=63, \bar{\omega}_{n_{d f}}=$ $95.46[\mathrm{rad} / \mathrm{s}], \bar{\zeta}=0.966$ and $\omega_{c_{d f}}=9.71[\mathrm{rad} / \mathrm{s}] ;\left|\mathrm{G}_{\mathrm{HPF}_{d f}}(0)\right|=\epsilon=$ $1 / 50=-34 \mathrm{db}$ and $\left|\mathrm{G}_{\mathrm{HPF}_{d f}}(\infty)\right|=1$.

\section{TRAJECTORY TRACKING}

In this section we present outdoor real-time experiments obtained when the quadrotor tracks a circular trajectory in the $x-y$ plane. For this:

1) The quadrotor is locally stabilized with the LQR of Section II-B, and robustly linearized with the drift-free estimators of Section III.

2) An optimal state trajectory is synthesized for reaching a given local stationary point.

3) The circular trajectory is partitioned by a finite set of suitable local stationary points.

\section{A. Control Scheme}

In [3], we have shown that the nonlinear uncertainty signal estimator (2.19), has two interesting properties: it enlarges the stability neighborhood and it creates an attraction neighborhood $\mathcal{B}_{\rho}$, of radius $\underline{\rho}$, around the stationary point (see Figure 3 of $\left.{ }^{* *}[3]\right)$.

\footnotetext{
${ }^{7}$ Doing: $\left|\mathrm{G}_{\mathrm{HPF}_{d f}}(\jmath \omega)\right|=1$, one gets: $2 a_{o, 4} \omega^{4}-2 a_{o, 2} a_{o, 4} \omega^{2}+(1+$ e) $a_{o, 4}^{2}=0$.
}

In consequence, we have partitioned the desired trajectory for tracking, $\mathbf{x}_{i}^{*}(t)(i \in\{\mathrm{x}, \mathrm{y}\}), t \in\left[0, T_{f}\right]$, by a set of $N+1$ stationary points:

$$
\Lambda_{S P_{i}}^{*}=\left\{\overline{\mathbf{x}}_{0, i}^{*}, \overline{\mathbf{x}}_{1, i}^{*}, \ldots, \overline{\mathbf{x}}_{N, i}^{*}\right\}, \quad \mathbf{x}_{i}^{*}\left(k T_{s}\right)=\overline{\mathbf{x}}_{k, i}^{*},
$$

where: $k \in\{0,1, \ldots, N\}, N T_{s}=T_{f}$ and $i \in\{\mathrm{x}, \mathrm{y}\}$; $T_{s}$ is the trajectory sampling time and $T_{f}$ is the flying time. Then, the local stabilizing feedbacks (2.11) and the drift-free estimators (3.3) take the forms, respectively:

$$
\begin{aligned}
& \mathbf{u}_{i}=\mathbf{F}_{i}\left(\mathbf{x}_{i}-\mathbf{x}_{i}^{*}\right)+\overline{\mathbf{u}}_{d f, i}, \\
\frac{\mathrm{d}}{\mathrm{d} t} \mathbf{w}_{d f, i}= & \left(\mathbf{A}_{\mathbf{K}_{i}}+(1-\epsilon) \mathbf{B}_{i} \mathbf{G}_{i}^{\ell} \mathbf{C}_{i}\right) \mathbf{w}_{d f, i} \\
& -\left(\mathbf{K}_{i}+(1-\epsilon) \mathbf{B}_{i} \mathbf{G}_{i}^{\ell}\right) \mathbf{C}_{i}\left(\mathbf{x}_{i}-\mathbf{x}_{i}^{*}\right), \\
\overline{\mathbf{u}}_{d f, i}= & (1-\epsilon) \mathbf{G}_{i}^{\ell}\left(\mathbf{C}_{i} \mathbf{w}_{d f, i}-\mathbf{C}_{i}\left(\mathbf{x}_{i}-\mathbf{x}_{i}^{*}\right)\right),
\end{aligned}
$$

where $i \in\{\mathrm{x}, \mathrm{y}, \psi\}$, and together with (2.13), (2.20), (2.32) and $\epsilon=1 / 50^{8}$.

\section{B. Trajectory Planning}

1. Optimal state trajectory: In order to synthesize the optimal state trajectories which reach a given local stationary point, let us consider the linear state space descriptions ( $c f$. (2.2), (2.3), (2.11) and (2.13)):

$$
\mathrm{d} \mathbf{x}_{i}^{*} / \mathrm{d} t=\mathbf{A}_{\mathbf{F}_{i}} \mathbf{x}_{i}^{*}+\mathbf{B}_{i} \overline{\mathbf{u}}_{i}^{*}, \quad i \in\{\mathrm{x}, \mathrm{y}\},
$$

where: $\mathbf{A}_{\mathbf{F}_{i}} \doteq \mathbf{A}_{i}+\mathbf{B}_{i} \mathbf{F}_{i}, i \in\{\mathbf{x}, \mathbf{y}\}$, and with initial conditions: $\mathbf{x}_{i}^{*}(0)=\mathbf{x}_{o, i}$; let us note that the pairs $\left(\mathbf{A}_{\mathbf{F}_{i}}, \mathbf{B}_{i}\right)$ are controllable $(c f .(2.3))$.

We are interested in finding a minimal norm control input $\overline{\mathbf{u}}_{i}^{*}$, such that: $\mathbf{x}_{i}^{*}\left(T_{s}\right)=0$, where $T_{s}$ is a given finite time, $T_{s}>0$.

This is a classical minimum norm problem of seeking the closest vector to the origin lying in a variety of finite codimension, in a Hilbert space, and it is solved with the help of the Projection Theorem. Indeed, according to Theorem 2 of Section 3.3 of [7], the control input $\overline{\mathbf{u}}_{i}^{*}$ solving the problem has the form (see also Theorem 1.1 of [9]):

$$
\overline{\mathbf{u}}_{i}^{*}(t)=-\mathcal{F}_{\mathbf{F}_{i}}\left(T_{s}-t\right) \mathfrak{W}_{i_{T_{s}}}^{-1} \exp \left(\mathbf{A}_{\mathbf{F}_{i}} T_{s}\right) \mathbf{x}_{o, i},
$$

$i \in\{\mathrm{x}, \mathrm{y}\}, t \in\left[0, T_{s}\right]$, where: ${ }^{9}$

$$
\begin{aligned}
& \mathcal{F}_{\mathbf{F}_{i}}(t)=\mathbf{B}_{i}^{T} \exp \left(\mathbf{A}_{\mathbf{F}_{i}}^{T} t\right), \\
& \mathfrak{W}_{i_{T_{s}}}=\int_{0}^{T_{s}} \mathcal{F}_{\mathbf{F}_{i}}^{T}\left(T_{s}-\tau\right) \mathcal{F}_{\mathbf{F}_{i}}\left(T_{s}-\tau\right) \mathrm{d} \tau .
\end{aligned}
$$

From (4.16), (4.17) and (4.18), we get for all the time intervals, $\left[k T_{s},(k+1) T_{s}\right), k \in\{0,1, \ldots,(N-1)\}$, the following optimal trajectories:

$$
\begin{aligned}
\mathbf{x}_{i}^{*}(t)= & \exp \left(\mathbf{A}_{\mathbf{F}_{i}}\left(t-k T_{s}\right)\right) \overline{\mathbf{x}}_{k, i}^{*} \\
& +\int_{k T_{s}}^{t} \mathcal{F}_{\mathbf{F}_{i}}^{T}(t-\tau) \boldsymbol{\beta}_{i}^{*}(t, \tau) \mathbf{v}_{k, i}^{*} \mathrm{~d} \tau,
\end{aligned}
$$

${ }^{8}$ For the $\psi$ dynamics, we have chosen: $\mathbf{x}_{\psi}^{*}=0, \mathbf{A}_{\mathbf{F}_{\psi}}=$ $\left[\begin{array}{cc}0 & -a_{\psi, 2} \\ 1 & -a_{\psi, 1}\end{array}\right], \mathbf{B}_{\psi}=\left[\begin{array}{c}1 / \mathcal{I}_{z z} \\ 0\end{array}\right], \mathbf{K}_{\psi}=\left[\begin{array}{c}a_{\psi, 2}-a_{o, 2} \\ a_{\psi, 1}-a_{o, 1}\end{array}\right], \mathbf{C}_{\psi}$ $=\left[\begin{array}{ll}0 & 1\end{array}\right], \mathbf{G}_{\psi}^{\ell}=-\left(\mathbf{C}_{\psi}\left(\mathbf{A}_{\mathbf{F}_{\psi}}+\mathbf{K}_{\psi}^{o} \mathbf{C}_{\psi}\right)^{-1} \mathbf{B}_{\psi}\right)^{\ell}=a_{o, 2} \mathcal{I}_{z z}$, where: $\pi_{e}(\mathrm{~s})=\mathrm{s}^{2}+a_{o, 1} \mathrm{~s}+a_{o, 2}=\mathrm{s}^{2}+208 \mathrm{~s}+2704$, and $\epsilon=1 / 10$.

${ }^{9}$ Since the pairs $\left(\mathbf{A}_{\mathbf{F}_{i}}, \mathbf{B}_{i}\right)$ are controllable, the matrices $\mathfrak{W}_{i_{T_{s}}}$ are invertible [9]. 


$$
\begin{aligned}
\boldsymbol{\beta}_{i}^{*}(t, \tau) & =\mathcal{F}_{\mathbf{F}_{i}}(t-\tau) \mathfrak{W}_{i_{T_{s}}}^{-1} \\
\mathbf{v}_{k, i}^{*} & =\mathbf{x}_{(k+1), i}^{*}-\exp \left(\mathbf{A}_{\mathbf{F}_{i}} T_{s}\right) \overline{\mathbf{x}}_{k, i}^{*},
\end{aligned}
$$

where: $\overline{\mathbf{x}}_{k, i}^{*} \in \Lambda_{S P_{i}}^{*}, i \in\{\mathrm{x}, \mathrm{y}\}$ (recall (4.13)).

2. Stationary points set: We are interested in following the circular trajectory:

$$
\mathrm{x}_{*}^{2}(t)+\left(\mathrm{y}_{*}(t)-\boldsymbol{r}_{*}\right)^{2}=\boldsymbol{r}_{*}^{2},
$$

namely:

$$
\mathrm{x}_{*}(t)=\boldsymbol{r}_{*} \sin \left(\boldsymbol{\omega}_{s} t\right) \quad \text { and } \quad \mathrm{y}_{*}(t)=\boldsymbol{r}_{*}\left(1-\cos \left(\boldsymbol{\omega}_{s} t\right)\right),
$$

where: $\boldsymbol{\omega}_{s}=2 \pi / T_{f}$. Partitioning $T_{f}$, in $N$ points we get $\left(t=(k / N) T_{f}, k \in\{0,1, \ldots, N\}\right):{ }^{10}$

$$
\begin{aligned}
& \left(\overline{\mathbf{x}}_{k, \mathrm{x}}^{*}, \overline{\mathbf{x}}_{k, \mathrm{y}}^{*}\right)=\boldsymbol{r}_{*}( \\
& {\left[\begin{array}{llll}
\sin \left(\boldsymbol{\alpha}_{k}\right) & \boldsymbol{\omega}_{s} \cos \left(\boldsymbol{\alpha}_{k}\right) & \frac{\boldsymbol{\omega}_{s}^{2}}{g} \sin \left(\boldsymbol{\alpha}_{k}\right) & \frac{\boldsymbol{\omega}_{s}^{3}}{g} \cos \left(\boldsymbol{\alpha}_{k}\right)
\end{array}\right]^{T},} \\
& {\left[\begin{array}{lll}
\left(1-\cos \left(\boldsymbol{\alpha}_{k}\right)\right) & \boldsymbol{\omega}_{s} \sin \left(\boldsymbol{\alpha}_{k}\right) & \frac{\boldsymbol{\omega}_{s}^{2}}{g} \cos \left(\boldsymbol{\alpha}_{k}\right)
\end{array}\right.} \\
& \left.\left.-\frac{\boldsymbol{\omega}_{s}^{3}}{g} \sin \left(\boldsymbol{\alpha}_{k}\right)\right]_{(4.23)}^{T}\right)
\end{aligned}
$$

where: $\boldsymbol{\alpha}_{k}=2 \pi k / N$.

\section{Outdoor real-time experiments}

In this Section we present outdoor real-time experiments, when tracking a circular trajectory of a radius of $5[\mathrm{~m}]$, in the $x-y$ plane. For this, we have proceeded as follows:

- We have locally stabilized the Quadrotor with the state feedbacks (4.14) (for $i \in\{\mathrm{x}, \mathrm{y}, \mathrm{z}\}$ ) and (2.12) (for $i=$ z), together with (2.13).

- We have robustly linearized the quadrotor with the driftfree estimators (4.15) (for $i \in\{\mathrm{x}, \mathrm{y}, \mathrm{z}\}$ ), together with (2.20), (2.32) and ${ }^{11} \epsilon=1 / 50$.

- The circular trajectory for tracking was generated with the help of (4.19), (4.20), (4.18) and (4.23), with: $\boldsymbol{r}_{*}=$ $5[\mathrm{~m}]$ and $T_{s}=1[\mathrm{~s}]($ recall $(2.3)$ and $(2.13)){ }^{12}$

We have tracked the circular trajectory (4.21) with the radius $\boldsymbol{r}_{*}=5[\mathrm{~m}]$, a sampling time of $T_{s}=1[\mathrm{~s}]$, and a partition of $N=20$ points, namely: $T_{f}=20$ [s]. In Figure 3 , we show the obtained results when applying the local stabilizing feedbacks (4.14), with and without the drift-free estimators (4.15). We compare the obtained path v.s. the goal path (4.21), and the quadrotor (x, y)-trajectories v.s. the references (4.22). We also show the tracking error,

$$
e_{\mathcal{C}}(t)=\sqrt{\left(\mathrm{x}(t)-\mathrm{x}_{*}(t)\right)^{2}+\left(\mathrm{y}(t)-\mathrm{y}_{*}(t)\right)^{2}},
$$

when applying the local stabilizing feedbacks (4.14) together with the drift-free estimators (4.15), $i \in\{\mathrm{x}, \mathrm{y}\}$, we have

\footnotetext{
${ }^{10}$ From (2.3) and (4.16), we have: $\mathbf{x}_{2, x}^{*}=\mathrm{d} \mathbf{x}_{1, x}^{*} / \mathrm{d} t, \mathbf{x}_{3, \mathrm{x}}^{*}=$ $-g^{-1} \mathrm{~d} \mathbf{x}_{2, \mathrm{x}}^{*} / \mathrm{d} t, \quad \mathbf{x}_{4, \mathrm{x}}^{*}=\mathrm{d} \mathbf{x}_{3, \mathrm{x}}^{*} / \mathrm{d} t, \quad \mathbf{x}_{2, \mathrm{y}}^{*}=\mathrm{d} \mathbf{x}_{1, y}^{*} / \mathrm{d} t, \quad \mathbf{x}_{3, y}^{*}=$ $g^{-1} \mathrm{~d} \mathbf{x}_{2, \mathrm{y}}^{*} / \mathrm{d} t, \mathbf{x}_{4, \mathrm{y}}^{*}=\mathrm{d} \mathbf{x}_{3, y}^{*} / \mathrm{d} t$.

${ }^{11}$ Recall footnote 8 .

${ }^{12}$ In, we give details on the numerical aspects of (4.19) and (4.20).
}

obtained the maximum peak error: $\left\|e_{c}\right\|_{p}=\mathbf{0 . 5 4 6 3} \mathrm{m}$ and the root mean square error: $\left\|e_{c}\right\|_{r m s}=0.2992 \mathrm{~m}$, where:

$$
\left\|e_{\mathcal{C}}\right\|_{p}=\max _{t \in\left[0, T_{f}\right]} e_{\mathcal{C}}(t), \quad\left\|e_{\mathcal{C}}\right\|_{r m s}=\sqrt{\frac{1}{T_{f}} \int_{0}^{T_{f}} e_{\mathcal{C}}^{2}(t) \mathrm{d} t} .
$$

While, when applying only the local stabilizing feedbacks (4.14), $i \in\{\mathrm{x}, \mathrm{y}\}$, we have obtained the maximum peak

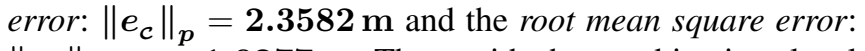
$\left\|e_{c}\right\|_{r m s}=1.0277 \mathrm{~m}$. Thus, with the combination, local stabilizing feedbacks (4.14) and drift-free estimators (4.15), we get the efficiencies: $\mathfrak{e}_{\mathfrak{f f}} p=\mathbf{8 9 . 0 7 4} \%$ and $\mathfrak{e}_{\mathfrak{f f}} \boldsymbol{r}_{\boldsymbol{r} m \boldsymbol{s}}=$ 91.537\%, and with only the local stabilizing feedbacks (4.14), we get the efficiencies: $\mathfrak{e}_{\mathfrak{f f}} \mathbf{p}=\mathbf{5 2 . 8 3 6} \%$ and $\mathfrak{e}_{\mathfrak{f f}} \mathbf{r m s}$ $=\mathbf{7 0 . 9 3 2} \%$, where: $^{13}$

$$
\begin{aligned}
\mathfrak{e}_{\mathfrak{f f}_{p}} & =\left(1-\left\|e_{\mathcal{C}}\right\|_{p} / \boldsymbol{r}_{*}\right) \times 100 \% \\
\mathfrak{e}_{\mathfrak{f f}_{r m s}} & =\left(1-\sqrt{2}\left\|e_{\mathcal{C}}\right\|_{r m s} / \boldsymbol{r}_{*}\right) \times 100 \% .
\end{aligned}
$$

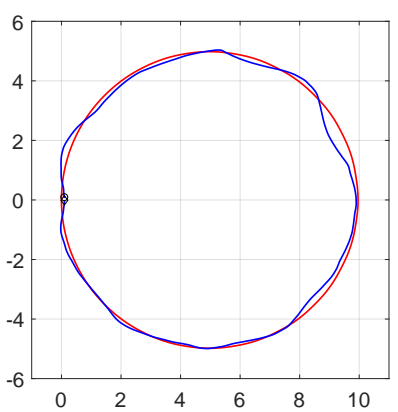

(a)
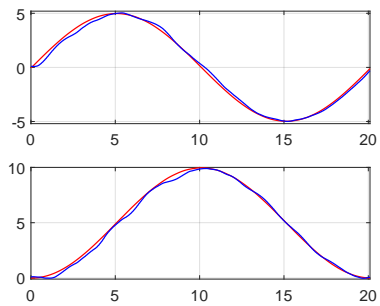

(c)

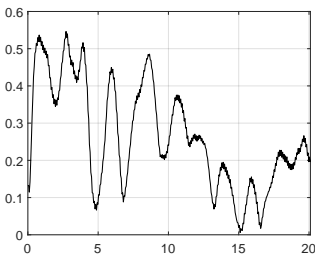

(e)

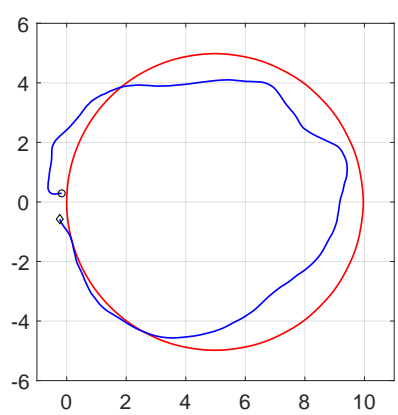

(b)
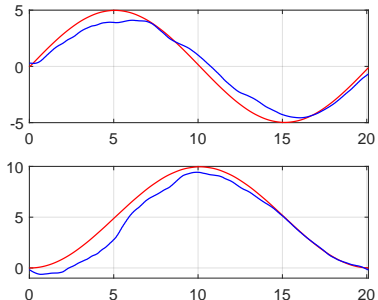

(d)

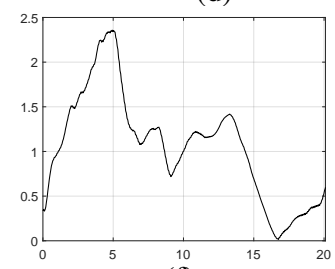

(f)
Fig. 3. Tracking of a circular trajectory in the plane $x-y$ with a $5[\mathrm{~m}]$ radius in $20[\mathrm{~s}]\left(N=20\right.$ and $\left.T_{s}=1[\mathrm{~s}]\right) .(\mathrm{a}, \mathrm{b}) \times[\mathrm{m}]$ v.s. y $[\mathrm{m}] .(\mathrm{c}, \mathrm{d})$ $\mathrm{x}[\mathrm{m}]$ v.s. $t[\mathrm{~s}]$ and $\mathrm{y}[\mathrm{m}]$ v.s. $t[\mathrm{~s}] .(\mathrm{e}, \mathrm{f})\left\|e_{\mathcal{C}}(t)\right\|[\mathrm{m}]$ v.s. $t[\mathrm{~s}]$. Applying the local stabilizing feedbacks (4.14) together with the drift-free estimators (4.15): (a) and (c). Applying only the local stabilizing feedbacks (4.14): (b) and (d). (a, b) Comparison of the obtained path v.s. the goal path (4.21). (c, d) Tracking error $e_{\mathcal{C}}(t)$ (see (4.24)).

The quadrotor was flown at an altitude around ${ }^{14} 3$ and 6 [m]; see Figure 4.

\footnotetext{
${ }^{13}$ Recall that: $\sqrt{\left(1 / T_{f}\right) \int_{0}^{T_{f}} \sin ^{2}\left(2 \pi t / T_{f}\right) \mathrm{d} t}=1 / \sqrt{2}$.

${ }^{14}$ This altitude was visually fixed with the only purpose of having a good sight.
} 


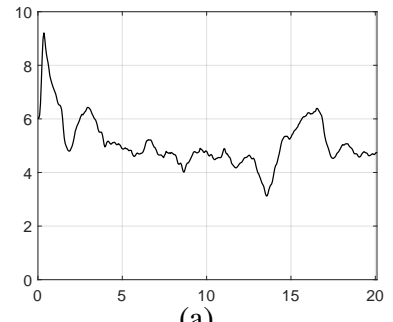

(a)

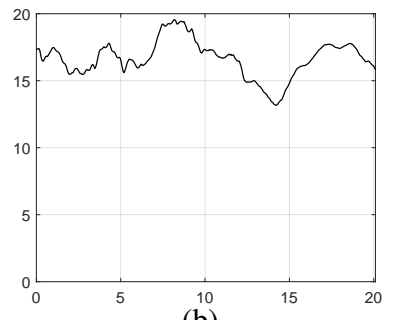

(b)
Fig. 4. Yaw trajectories $\psi\left[^{\circ}\right]$ v.s. $t[\mathrm{~s}]$. (a) Applying (4.14) and (4.15). (b) Applying only (4.14): (b) and.

From these outdoor real-time experiments, we see that for the slow trajectory we have obtained an efficiency improvement around $10 \%$, when applying the drift-free estimators (4.15); and for the fast trajectory the efficiency improvement was around $20 \%$. Let us also note that the yaw error diminishes by a $1 / 3$ factor, when applying the drift-free estimator (4.15); see Figure 4.

\section{CONCLUSION}

In this paper, we have tackled the problem of tracking some prescribed trajectory in the $x-y$ plane. We have found that there exists a drift phenomenon due to the wind noise. In Section III, we have characterized the disturbance wind (see (3.1)), and we have checked that when the term (3.2), passes trough the integral action of the nonlinear uncertainty estimator (2.19), such a drift phenomenon occurs. Depending on the values of the scaling factors of (3.1) this drift phenomenon could arise, and when arising the nonlinear uncertainty signal estimator (2.19) will not have a good performance. Thus, we have to do some adjustments to the nonlinear uncertainty signal estimator (2.19) in order to ensure a good performance not mattering the scale factors of (3.1).

To overcome the quadrotor drift due to the integration of wind disturbance like (3.2), we have proceeded as in [4], [5] shifting the pole at the origin of (2.19) slightly into the left-half complex plane, having now the drift-free estimator (3.3); see also the comparison shown in Figure 2.

We have done outdoor real-time experiments obtained when the quadrotor tracks a circular trajectory of a radius of $5[\mathrm{~m}]$ in the $\mathrm{x}-\mathrm{y}$ plane; we have first tracked a $5[\mathrm{~m}]$ radius circular trajectory in $60[\mathrm{~s}]$ (slow trajectory), and then a $5[\mathrm{~m}]$ radius circular trajectory in 20 [s] (fast trajectory). In Section IV, we are showing the obtained results for the fast trajectory case. From these experiences we got an apreciable reduction on the tracking and yaw errors when applying the drift-free estimators (4.15). We have obtained an efficiency improvement around of the $10 \%$, in the slow trajectory experience, and an efficiency improvement around of the $20 \%$, in the fast trajectory experience, see Figure 3; with respect to the yaw error, it decreased by a $1 / 3$ factor.

\section{REFERENCES}

[1] Blas, L.A., Bonilla, M., Malabre, M., Azhmyakov, V. and Salazar, S. (2017). Structural feedback linearization based on nonlinearities rejec- tion. IFAC-World Congress, pp. 945-950. July 9 - 14, 2017. Toulouse, France.

[2] Blas, L.A., Bonilla, M.,Salazar, S, Malabre, M. and Azhmyakov, V. (2019). Synthesis of a robust linear structural feedback linearization scheme for an experimental quadrotor. 18th European Control Conference, pp. 1431-1436. June 25-28, 2019 . Napoli, Italy.

[3] Bonilla, M., Blas, L.A., Azhmyakov, V., Malabre, M. and Salazar, S. (2020). Robust structural feedback linearization based on the nonlinearities rejection. Journal of the Franklin Institute 357, pp 2232-2262. https://doi.org/10.1016/j.jfranklin.2019.11.044.

[4] Gavin, H.P., Morales, R., and Reilly, K. (1998). Drift-free integrators. Review of Scientific Instruments 69(5), May, 2171-2175.

[5] Horowitz, P. and Hill, W. (1989). The Art of Electronics, 2nd ed. Cambridge University Press, Cambridge.

[6] Kailath, Th. (1980). Linear systems, Prentice-Hall, New Jersey.

[7] Luenberger D.G. (1969). Optimization by Vector Space Methods, John Wiley \& Sons, Inc., New York.

[8] Vidyasagar M. (1993). Nonlinear Systems Analysis, 2nd edition Prentice-Hall, Inc. New Jersey.

[9] Wonham, W.M. Linear Multivariable Control: A Geometric Approach, New York: Springer-Verlag, 3rd ed, 1985.

\section{APPENDIX}

For synthesizing the optimal state trajectory given by (4.19) and (4.20), we have first applied the change of bases: $\left(\overline{\mathbf{A}}_{\mathbf{F}_{i}}, \overline{\mathbf{B}}_{i}\right)=\left(\mathbf{T}_{i}^{-1} \mathbf{A}_{\mathbf{F}_{i}} \mathbf{T}_{i}, \mathbf{T}_{i}^{-1} \mathbf{B}_{i}\right), i \in\{\mathrm{x}, \mathrm{y}\}$, in order to simplify the exponential matrix computation, where: $\mathbf{T}_{\times}^{-1}=\left[\begin{array}{cccc}0 & 626.5 & -3216.0 & 0 \\ 0 & -1.205 & 0.003678 & 0 \\ 1 & 0.5236 & -0.001597 & 0 \\ 0 & -626.1 & 3216.0 & 1\end{array}\right]$ and $\mathbf{T}_{\mathrm{y}}^{-1}=$
$\left[\begin{array}{cccc}0 & 654.5 & 3360.0 & 0 \\ 0 & 1.205 & 0.00352 & 0 \\ 1 & 0.5236 & 0.001529 & 0 \\ 0 & 654.2 & 3360 & 1\end{array}\right] .{ }^{2} .5$

1) The convolution integrals of (4.19) were synthesized with the discretization of the state space descriptions $\Sigma\left(\overline{\mathbf{A}}_{\mathbf{F}_{i}}, \overline{\mathbf{B}}_{i}\right)$, $i \in\{\mathrm{x}, \mathrm{y}\}$, in the time interval $\left[0, T_{s}\right), T_{s}=1$, and with the initial conditions (4.23).

2) The exponential matrices of (4.19) are: $\exp \left(\mathbf{A}_{\mathbf{F}_{\times}} T_{s}\right)=\mathbf{T}_{\times} \exp \left(\overline{\mathbf{A}}_{\mathbf{F}_{\times}} T_{s}\right) \mathbf{T}_{\times}^{-1}=$

$$
\begin{aligned}
& {\left[\begin{array}{llll}
0.6118 & 0.4581 & -1.1060 & -0.0003
\end{array}\right]} \\
& \left.\begin{array}{llll}
-0.7364 & -0.1841 & -0.2737 & -0.0001
\end{array}\right] \\
& \begin{array}{llll}
0.0186 & 0.0952 & -0.2909 & -0.0001
\end{array} \\
& {\left[\begin{array}{llll}
-0.1938 & -0.1908 & 0.1764 & 0.0001
\end{array}\right]} \\
& \exp \left(\mathbf{A}_{\mathbf{F}_{\mathbf{y}}} T_{s}\right)=\mathbf{T}_{\mathbf{y}} \exp \left(\overline{\mathbf{A}}_{\mathbf{F}_{\mathbf{y}}} T_{s}\right) \mathbf{T}_{\mathrm{y}}^{-1}= \\
& {\left[\begin{array}{rrrr}
0.6118 & 0.4581 & 1.1060 & 0.0003 \\
-0.7364 & -0.1841 & 0.2737 & 0.0001 \\
-0.0186 & -0.0952 & -0.2909 & -0.0001 \\
0.1938 & 0.1908 & 0.1764 & 0.0001
\end{array}\right] \text {. }}
\end{aligned}
$$

and:

3) The $\boldsymbol{\beta}_{i}^{*}(t, \tau)$ are: $\boldsymbol{\beta}_{\times}^{*}(t, \tau)=\mathcal{F}_{\mathbf{F}_{\times}}(t-\tau) \mathfrak{W}_{\mathrm{x}_{s}}^{-1} \mathbf{T}_{\times}^{-1}$

$=k_{1, \times} \mathrm{e}^{a_{1, \times}(t-1)}+k_{2, \times} \mathrm{e}^{a_{2, \times}(t-1)}+\left(k_{3, \times} \cos \left(\omega_{\mathrm{x}}(t-1)\right)\right.$

$\left.+k_{4, \mathrm{x}} \sin \left(\omega_{\mathrm{x}}(t-1)\right)\right) \mathrm{e}^{b_{\mathrm{x}}(t-1)}$ and $\boldsymbol{\beta}_{\mathrm{y}}^{*}(t, \tau)=\mathcal{F}_{\mathbf{F}_{\mathrm{y}}}(t-$

$\tau) \mathfrak{W}_{\mathrm{y}_{T_{s}}}^{-1} \mathbf{T}_{\mathrm{y}}^{-1}=k_{1, \mathrm{y}} \mathrm{e}^{a_{1, \mathrm{y}}(t-1)}+k_{2, \mathrm{y}} \mathrm{e}^{a_{2, \mathrm{y}}(t-1)}+$

$\left(k_{3, y} \cos \left(\omega_{\mathrm{y}}(t-1)\right)+k_{4, \mathrm{y}} \sin \left(\omega_{\mathrm{y}}(t-1)\right)\right) \mathrm{e}^{b_{\mathrm{y}}(t-1)}$, where: $a_{1, \mathrm{x}}=3214, a_{2, \mathrm{x}}=1.911, \omega_{\mathrm{x}}=1.585, b_{\mathrm{x}}=0.9536$, $a_{1, \mathrm{y}}=3358, a_{2, \mathrm{y}}=1.911, \omega_{\mathrm{y}}=1.585, b_{\mathrm{y}}=0.9536$, $\left[k_{1, \times} k_{2, \mathrm{x}} k_{3, \times} k_{4, \mathrm{x}}\right]=$

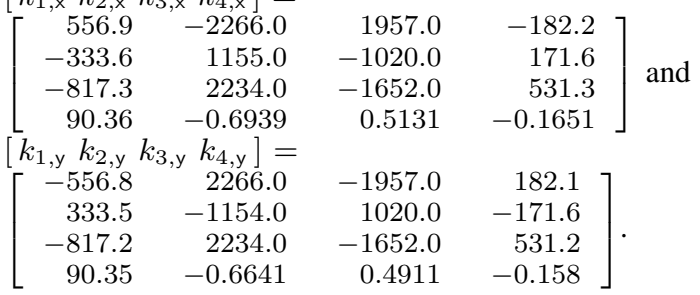

${ }^{15}$ In this section we present all the numerical values with four significant digits, but in the outdoor real-time experiments we have used eight significant digits. 